\title{
Topical Application of Thermobalancing Therapy Enabled by Therapeutic Device Treats Enlarged Prostate and Improves Men's Quality of Life; New Opportunity in BPH Treatment
}

\author{
Simon Allen ${ }^{1 *}$ and Ivan Gerasimovich Aghajanyan ${ }^{2}$ \\ ${ }^{1}$ Allen S, Director of Fine Treatment, 29 Rewley Road, Oxford, OX1 2RA, UK \\ ${ }^{2}$ Aghajanyan IG, Head of the Department of Urology at the Yerevan State Medical University, Republic of Armenia, 9 Ezras Hasratyan St, Yerevan, 0052, \\ Armenia, UK
}

Received: February 15, 2016; Accepted: April 23, 2016; Published: April 26, 2016

*Corresponding author: Allen Simon, Director of Fine Treatment, 29 Rewley Road, Oxford, OX1 2RA, United Kingdom; Tel: +447958878300; Fax: +441865728255; E-mail:info@finetreatment.com

\begin{abstract}
Background: Benign Prostatic Hyperplasia (BPH) is the most common problem for men over 50 that causes Lower Urinary Tract Symptoms (LUTS) and is usually treated with phytotherapy, medications and surgical procedures. The aim of our study was to find out if topically applied therapeutic device would be effective for LUTS due to $\mathrm{BPH}$

Methods: 124 men older than 55 with BPH, with prostate size less than $60 \mathrm{~mL}$, used therapeutic device by applying a natural thermo element to the projection of prostate during 6 months. 124 men with BPH in the control group didn't receive therapy. Before and after the 6-month period were investigated the International Prostate Symptom Score (IPSS), Quality Of Life (QoL) index, Prostate Volume (PV) by ultrasound and maximum urinary flow rate $\left(\mathrm{Q}_{\max }\right)$ by uroflowmetry. In this article we also present changes of the clinical symptoms and parameters in men with BPH, with PV > $60 \mathrm{~mL}$ under Thermobalancing therapy.
\end{abstract}

Results: After 6-month application of the device as mono therapy QoL improved, reduced IPSS $(P<0.001)$ and PV $(P<0.001)$, and increased $Q_{\max }(P<0.001)$. The dynamics of the same measurements in the control-group have shown negative outcomes. The positive outcomes were also recorded in men with $\mathrm{BPH}$, with $\mathrm{PV}>60 \mathrm{~mL}$.

Conclusions: For the first time a topical treatment was used for men with BPH. Thermobalancing therapy, enabled by therapeutic device, reduced PV and urinary symptoms, improved QoL and increased $Q_{\max }$ gradually and naturally. Thus, a topically applied treatment can be recommended for men with LUTS due to prostate enlargement.

Keywords: BPH treatment; Prostate enlargement; Thermobalancing therapy; Prostate natural treatment; Lower urinary tract symptoms; LUTS; Benign prostatic hyperplasia; Enlarged prostate; Clinical trial

\section{Background}

The frequency of Lower Urinary Tract Symptoms (LUTS), as a result of Benign Prostatic Hyperplasia (BPH), increases with age and affect approximately 50\% of men over age 50. LUTS may include frequent urination, urgent urination, nocturia, urinary stream hesitancy, straining to void, and dribbling. LUTS/ BPH have a significant impact on men's quality of life [1].

Behavioral treatments and lifestyle modifications appear beneficial in all patients despite differences in the underlying pathophysiology. These options are rarely applied in routine practice but they are cheap and easy, therefore should be included in all treatment strategies. They include restriction of fluids a couple of hours before going to sleep, avoidance of caffeinated or alcoholic beverages in the evening, emptying the bladder before going to bed, leg elevation in case of edema of the lower extremities, moderate physical exercise and keeping warm in bed [2].

However, the focus of treatment for BPH/ LUTS has traditionally involved the use of alpha-blockers, $5 \alpha$-reductase inhibitors, and phytotherapy. A recent study revealed that improvements in QoL were equivalent across the medical treatments most widely used in real-life practice, such as alphaadrenergic blockers; 5-alpha-reductase inhibitors and combined treatment. Phytotherapy showed an equivalent efficacy to BPH medications with fewer side effects [3].

Approximately $30 \%$ of men diagnosed with prostate disease take supplements, and doctors in Europe typically use such remedies as first-line treatment for urologic conditions [4]. The most common for LUTS due to BPH are extracts of the saw palmetto. The recent data from double-blind clinical trials do not support efficacy superior to that of placebo, but the saw palmetto extract showed no evidence of toxicity [5]. Natural therapies have 
a long history of use in India to support optimal prostate health. Varuna, khadir, and punarnava have been shown to be effective for $\mathrm{BPH}$ [6]. And in Africa, for instance, in the University of Ghanathe study indicated that ethanolic root extract of $C$. membranaceus is one of the few monoherbal products that remarkably reduces PSA levels, prostate volume and subsequently improves the QoL of patients with BPH [7].

The German Research Activities on Natural Urological (GRANU) study was a randomized, partially blinded, placebocontrolled, parallel-group trial that investigated the efficacy of pumpkin seed in men with lower urinary tract symptoms suggestive of Benign Prostatic Hyperplasia (BPH/ LUTS) that concluded: Overall, in men with BPH, 12 months of treatment with pumpkin seed led to a clinically relevant reduction in IPSS compared with placebo [8].

There have been some researches which provide evidence for the clinical application of acupuncture for BPH. For instance, a treatment course of 3 months acupuncture achieves the significant efficacy on BPH, which is better than the oral administration of Qianliekang (Rape Flower Pollen) tablets [9]. Another study has evaluated the effects of Electroacupuncture (EA) stating that it is effective in improving patient's quality of life in men with BPH [10]. However, a single-blinded Randomized Controlled Trial (RCT) of acupuncture for BPH found no changes of prostate special antigen and international prostatic symptom score were not significantly different between two groups, suggesting the clinical improvement was quite limited to the LUTS of patients with BPH [11]. Thus, we see that almost all noninvasive methods for BPH/ LUTS treatment are associated with taking oral medications or natural remedies.

Dr. Allen's research on the Origin of Diseases has helped him to identify the causal root of prostate enlargement, namely capillary expansion. This conclusion is based on 2 functional physiological properties of capillaries which are activated by an irritating factor, i.e., a trigger; indeed this peculiarity of capillaries explains the cause of chronic internal diseases. Constriction of capillaries in response to an irritating trigger develops local micro-hypothermia. It is this focus of hypothermia, which in turn becomes a constant irritant maintaining illness, i.e., making a disease chronic [12].

In response to irritation (i.e., a trigger-initiator and later focus of hypothermia) and in order to eliminate them, the blood flow increases through the spontaneous expansion of the capillary net locally. The formation of new capillaries is essentially the growth of the excess tissue that leads to an increased pressure inside the organ and to the prostate growth. In order to terminate prostate growth and to reverse prostate enlargement, the improvement of blood circulation locally becomes the primary objective [13].

Therefore, in order to deliver targeted treatment to the prostate gland, topically applied therapeutic device that provides Thermobalancing therapy has been used in the study involving 124 men with BPH and age over 55, with the PV less $60 \mathrm{~mL}$. A 2-year controlled clinical trial has shown the efficacy of
Thermobalancing therapy for men with BPH [14]. In this study we present some documents of the clinical trial and also the dynamics of the clinical symptoms in a man with the prostate volume over $60 \mathrm{~mL}$, and also compare the natural treatment options for BPH.

\section{Materials and Methods}

\section{Study design}

The observational clinical controlled study was used. Enrolment began on April 2013 in the Department of Urology of the Yerevan State Medical University. The Ethics Committee of the Yerevan State Medical University has approved the clinical study on Thermobalancing therapy. Effectiveness of Thermobalancing therapy was studied by comparing men with BPH who received treatment with therapeutic device for 6-month with the control group on the watchful waiting stage. Dynamics of the symptoms and the indicators in each group were evaluated in comparison to their data in the beginning and end of the treatment.

\section{Evaluation}

The baseline evaluations included complete physical examination, medical history, DRE, serum biochemistry, and PSA measurements, electrolytes, urine and renal function tests. Evaluations were made at baseline and 6 months after the treatment. IPSS-QoL scored as follow: delighted $=0$, pleased $=$ 1 , mostly satisfied $=2$, about equally satisfied and dissatisfied $=3$, mostly dissatisfied $=4$, hopeless $=5$ and poor $=6$. PV was measured at baseline and at 6-month after the treatment by ultrasonography (US-9000E2 ultrasound scanner, Rising Medical Equipment Co. Ltd, Beijing, China) and uroflowmetry(maximum urinary flow rate $-Q_{\text {max }}, \mathrm{mL} / \mathrm{s}$ ) was used for the measurement of the rate of urine flow parameters (Sanuro2UL, Santron Meditronic, Maharashtra, India). The standard ellipsoid formula length $\times$ width $\times$ height $\times 0.52$ was used to determine prostate volume.

\section{Participants and interventions}

226 men were examined in total from April 2013 to December 2014 in total. 124 patients were selected for the clinical trial. Inclusion criteria: Men were eligible for enrollment if they were over the age of 55, in the absence of acute prostatitis, at the level of Prostate-Specific Antigen (PSA) not more than 4 m.mol/ l, $Q_{\text {max }}$ higher than $5 \mathrm{~mL} / \mathrm{sec}$. It did not matter if they were treated at the time of enrollment with medicines. However, after the use of therapeutic device other treatments were cancelled gradually. Exclusion criteria: $P V>60 \mathrm{ml}$, co-morbidities, such as diabetes, heart failure, cancer, etc.

Thus, 80 men were excluded, as their prostate volume was over $60 \mathrm{ml}$ or they had severe co-morbidities; 10 preferred operation; 4 were suspected prostate cancer; 8didnot attend to the following examinations. In total: 102 patients with BPH were excluded from the clinical trial.

In this article we also have presented changes of the clinical symptoms and parameters in 3 men with PV $>60 \mathrm{~mL}$ who used therapeutic device. Men in treatment-group after the screening 
Table 1: The changes in Prostate Volume (PV $\mathrm{mL}$ ) and the uroflowmetry (maximum urinary flow rate $\left(\mathrm{Q}_{\mathrm{max}} \mathrm{mL} / \mathrm{s}\right)$, Post Voiding Residual (ml) International Prostate Symptom Score (IPSS), in men with BPH (PV > $60 \mathrm{~mL}$ ) on Thermobalancing therapy.

\begin{tabular}{|c|c|c|}
\hline Galstyan L, age 75 & $03 / 2 / 2014$ & 03.11 .2014 \\
\hline IPSS - Urinary symptoms & 12 & 3 \\
\hline QoL score & 4 & 0 \\
\hline Prostate volume mL & 84 & 52 \\
\hline $\mathrm{Q}^{-}{ }_{\max } \mathrm{mL} / \mathrm{s}$ & 9.7 & 15.5 \\
\hline Sargsyan T, age 73 & 01/07/2014 & $03 / 11 / 2014$ \\
\hline IPSS - Urinary symptoms & 14 & 4 \\
\hline QoL score & 4 & 1 \\
\hline Prostate volume mL & 93 & 70 \\
\hline $\mathrm{Q}^{-{ }_{\max }} \mathrm{mL} / \mathrm{s}$ & 11.2 & 14.0 \\
\hline Nersesyan Telman age 79 & $22 / 07 / 2014$ & $18 / 02 / 2015$ \\
\hline IPSS & 6 & 2 \\
\hline QoL score & 3 & 1 \\
\hline Prostate volume mL & 154 & 90 \\
\hline $\mathrm{Q}_{\text {max }} \mathrm{mL} / \mathrm{s}$ & 13.0 & 14.4 \\
\hline
\end{tabular}

were given therapeutic device, termed Dr. Allen's Device, see figure 1.

\section{Statistical analysis}

Because Independent Samples t-Test and Pair Samples t-Test is only suitable for interval and ratio data, the Wilcoxon SignedRanks Test by using SPSS was conducted.

\section{Results}

\section{Urinary symptoms and QoL}

In the control group, the mean IPSS-UrS increased from 13.45 \pm 3.254 to $14.35 \pm 3.396$, whereas in the treatment group the mean IPSS-UrS decreased from $14.33 \pm 3.399$ to $4.73 \pm 2.754$ at the end of the observation period. For the control group, the $\mathrm{z}$ value was 6.018 with a $P$ value $(P<0.001)$. For the treatment group, the $\mathrm{z}$ value was 9.674 with a significance level $(P<0.001)$. This indicates that the treatment with Therapeutic Device decreased the urinary symptoms significantly, while in absence of treatment the symptoms increased significantly.

In the control group, the mean IPSS-QoL increases from 3.43 \pm 0.956 to $3.76 \pm 0.983$, whereas in the treatment group the mean IPSS-QoL decreases from $3.91 \pm 0.755$ to $1.39 \pm 1.110$. For the control group, the $\mathrm{z}$ value was 5.286 with a $P$ value $(P<0.001)$. For the treatment group, the $\mathrm{z}$ value was 9.672 with a $P$ value $(P<0.001)$. These results indicated that the treatment with Therapeutic device improved the QoL while in the control group the QoL worsened.

\section{Prostate volume and $\mathbf{Q}_{\max }$}

In the control group the mean prostate volume increased from 45.54 to $50.85 \mathrm{~mL}$, whereas in the treatment group the mean prostate volume decreased from 45.19 to $31.86 \mathrm{~mL}$. For the control group, the $\mathrm{z}$ value is -8.727 at the significance level $(P<0.001)$. So there was a statistically significant increase in

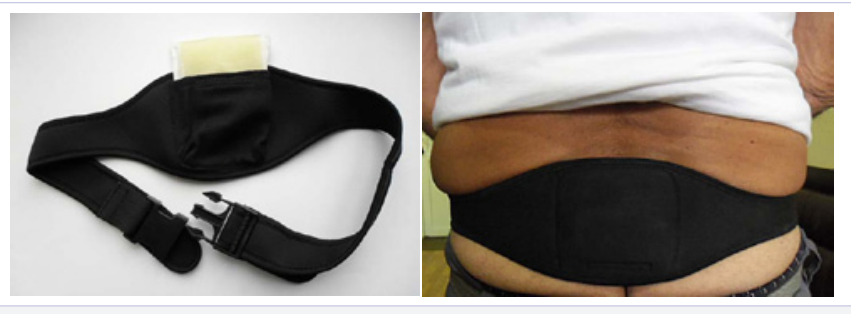

Figure 1: The therapeutic device alone (on the left) and the device tightly applied to the coccyx area in the projection of the prostate (on the right).

the prostate volume in the control group. For the treatment group, the $\mathrm{z}$ value is -9.669 at the significance level $(P<0.001)$. So the treatment with therapeutic device reduced the PV level significantly, whereas in the no treatment group the PV level increased.

In the control group, the mean $\mathrm{Q}_{\max }$ decreased from $7.95 \pm$ 2.871 to $7.7 \pm 2.695 \mathrm{~mL} / \mathrm{s}$, where as in the treatment group the mean $Q_{\max }$ increased from $8.10 \pm 3.041$ to $17.73 \pm 4.392 \mathrm{~mL} / \mathrm{s}$. For the control group, the $\mathrm{z}$ value was 1.929 and the $P$ value .054 $(>.05)$, indicating no statistically significant difference. For the treatment group, the $\mathrm{z}$ value is 9.621 at the significance level $(P$ $<0.001$ ), indicating a significant increase in the $Q_{\max }$. Therefore, our results demonstrate that the therapeutic device increased the uroflowmetry $Q_{\max }$ significantly in BHP patients, whereas the control group had no significant difference in the uroflowmetry $\mathrm{Q}_{\max }$.

\section{The clinical symptoms and parameters in men with BPH, PV > $60 \mathrm{~mL}$}

These cases show the use of the therapeutic device for patients with BPH (PV > $60 \mathrm{~mL}$ ) has led to improvement in IPSS, in both scores: urinary symptoms and QoL. Increased Q-max 


\section{The dynamics of clinical symptoms in men with BPH by I-PSS}

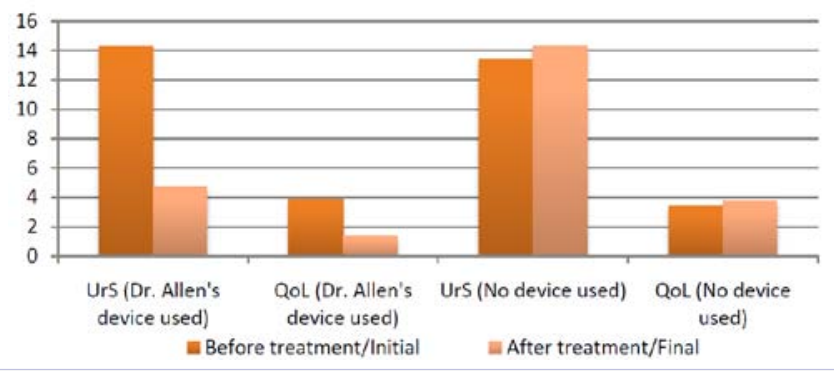

Figure 2: International Prostate Symptom Score (IPSS), urinary symptoms (UrS) and quality of life (QoL), in patients with BPH on Thermobalancing therapy and in the control group at the beginning and at the end of the study.

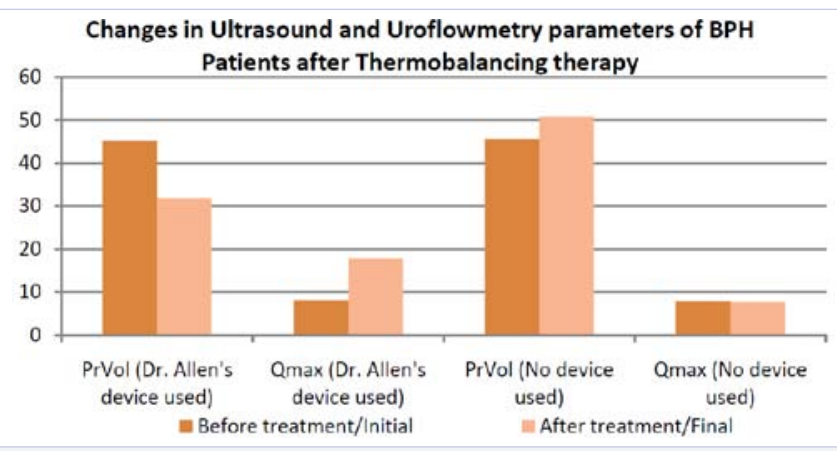

Figure 3: The changes in Prostate Volume (PV) $\mathrm{mL}$ and the uroflowmetry (maximum urinary flow rate $\left(Q_{\text {max }}, \mathrm{mL} / \mathrm{s}\right.$ ) in men with $\mathrm{BPH}$ on Thermobalancing therapy and in the control group at the beginning and at the end of the study.

after treatment was observed. The PV level decreased. However, at the end of the therapy the PV level was still high in these patients, $52 \mathrm{~mL}, 70 \mathrm{~mL}$ and $90 \mathrm{~mL}$ accordingly; therefore they were recommended to continue Thermobalancing therapy.

\section{Outcome measures}

Primary end points were the reduction of the IPSS and the increase of $Q_{\max }$ at 6-month after treatment. Secondary end points were the reduction of $\mathrm{PV}$, and changes in QoL at 6 months after treatment.

\section{Discussion}

This study results revealed that topical treatment for BPH/ LUTS was able to decrease IPSS and improve QoL significantly, reduce the PV level and increase the $Q_{\max }$. The large size of a prostate did not show to be obstruction for the use of therapeutic device, while for men with $Q_{\max }$ less $5 \mathrm{~mL} / \mathrm{s}$ were suggested surgical procedures. However, if for men with PV less than 60 $\mathrm{mL}$ the 6-month treatment period can be recommended, for men with larger prostate this time should be extended. The positive outcomes of this study indicate that Thermobalancing therapy is effective.

Widely used BPH medications cannot reach prostate and provide symptomatically relief in its acute stage. Moreover BPH drugs and prostate surgeries may be responsible for different side effects [15]. For instance, the case studies published by the Harvard Medical School confirm complications arising from two BPH drugs: tamsulosin (Flomax) which may suddenly make men become lightheaded or develop retrograde ejaculation, and terazosin (Hytrin) which can be responsible for the acute urinary retention [16]. Dr. Irwig has confirmed persistence of erectile dysfunction after discontinuation of treatment with Propecia (finasteride) that affects men's QoL. That is why a "watchful waiting" stage for BPH is recommended [17].

Regarding a role of complementary and alternative therapies in BPH management the view is based on facts that a 2012 systematic review of 32 RCTs involving 5,666 men treated for four to 72 weeks demonstrated that saw palmetto did not reduce nocturia, peak urine flow, prostate size, or AUA-SI scores compared with placebo in men with BPH. Systematic reviews of pygeum, cernilton, and herbs containing beta sitosterols found that trials were limited by short duration, small sample size, and varied doses and preparations. There is no current evidence to support their use [18].

As long-term BPH medications and herbal medicine don't protect men from the progress of BPH/ LUTS. Surgical treatment of prostate may also be accompanied serious complications. Thus, the results of a survey of sexually active men after three different laser surgeries from 2005 to 2010 concluded that these surgical techniques can have a negative impact on sexual function, and patients with normal preoperative sexuality are more at risk [19].

That is why EAU Guidelines on the Treatment and Followup of Non-neurogenic Male Lower Urinary Tract Symptoms Including Benign Prostatic Obstruction suggests: Prostate surgery is indicated in men with absolute indications or drug treatmentresistant LUTS due to benign prostatic obstruction [20].

Thermobalancing therapy can prevent the progress of BPH as it tackles its cause. In the last decade, the biological mechanism of BPH began to consider from the perspective of vascular dysfunction [21], chronic ischemic tissue [22], and increasing the pressure in the prostate gland [23]. The use of therapeutic device targets all aforementioned issues: capillaries, ischemia and the increased pressure, and that is why it is effective.

Thermobalancing therapy is entirely different than common heating treatments because it regulates the affected organ's temperature locally, maintaining it within the normal body temperature range. All other treatments with imposed heat can be damaging, because the high temperatures destroy delicate cells of the already stressed organ. For example, any temperature above 104 Fahrenheit $\left(\right.$ or $40^{\circ} \mathrm{C}$ ) can result in the death of the living organism. On the other side, low temperatures decrease cellular metabolism and, as a result, interfere with natural replenishment and healing.

Therapeutic device applies the thermo element topically tightly to the skin in the projection prostate that it overcomes the skin barrier spreading energy inside the body [24]. One may suggest that similar treatments have been available on the 
market for a long time (e.g. wool or synthetic fabric clothes) and that many patients with chronic pain wear such devices for a long time. This is not the case; however, as no other treatment method and material have been able to overcome the skin barrier delicately and precisely.

Thermobalancing therapy is the only external non-invasive treatment that targets pathological nidus continuously for a prolonged period of time, i.e., for days, months or even years, maintaining the accumulated body temperature in the project of a prostate [25]. We believe that the use of therapeutic device by keeping the temperature in the projection of the prostate gland acts on micro-focus of hypothermia and ischemia in it, removing the vicious cycle of spontaneous growth of capillaries in response to a trigger, micro-hypothermia, thereby reducing PV and relieving the BPH symptoms.

\section{Conclusions}

For the first time in the medical practice a natural topical treatment has been used for BPH/ LUTS. The results of the clinical trial have shown that Thermobalancing therapy enabled by therapeutic device provides effective and safe therapy improving men's QoL. This study has shown PV reduces despite of its initial size and the time period of using the device depends on PV, the larger prostate needs more time. Thus, we need more studies in order to establish correlation between PV and "time period of use the device". This study also suggests that Thermobalancing therapy, enabled by therapeutic device, can be offered to patients with BPH/ LUTS as a new effective treatment option without side effects.

\section{Safety and costs}

Patients feel no side effects during the clinical trial. Besides all of this, the important factor is the low price of a new treatment compared to the cost of the standard treatment options.

\section{Competing Interests}

The authors declare that they have no competing interests.

\section{References}

1. Gratzke C, Bachmann A, Descazeaud A, Drake MJ, Madersbacher S, Mamoulakis C, et al. EAU Guidelines on the Assessment of Nonneurogenic Male Lower Urinary Tract Symptoms including Benign Prostatic Obstruction. Eur Urol. 2015;67(6):1099-109.

2. Oelke M, Adler E, Marschall-Kehrel D, Herrmann TR, Berges R Nocturia: state of the art and critical analysis of current assessment and treatment strategies. World J Urol. 2014;32(5):1109-1117.

3. Alcaraz A, Carballido-Rodríguez J, Unda-Urzaiz M, Medina-López R, Ruiz-Cerdá JL, Rodríguez-Rubio F. Quality of life in patients with lower urinary tract symptoms associated with BPH: change over time in real-life practice according to treatment-the QUALIPROST study. Int Urol Nephrol. 2016;48(5):645-56. doi: 10.1007/s11255-015-1206-7.

4. Jackson CB, Taubenberger SP, Botelho E, et al. Complementary and Alternative Therapies for Urinary Symptoms: Use in a diverse population sample qualitative study, Urol Nurs. 2012;32(3):149-157.

5. Avins AL, Lee JY, Meyers CM, Barry MJ, CAMUS Study Group. Safety and Toxicity of Saw palmetto in the Complementary and
Alternative Medicine for Urological Symptoms (CAMUS) Trial J Urol. 2013;189(4):1415-1420.

6. Shrivastava A, Gupta VB. Various treatment options for benign prostatic hyperplasia: A current update. J Midlife Health. 2012;3(1):10-19.

7. Asare GA, Afriyie D, Ngala RA, Appiah AA, Anang Y, Musah I, et al. Shrinkage of Prostate and Improved Quality of Life: Management of BPH Patients with Croton membranaceus Ethanolic Root Extract. Evid Based Complement Alternat Med. 2015;2015:365205.

8. Vahlensieck W, Theurer C, Pfitzer E, Patz B, Banik N, Engelmann U, et al. Effects of Pumpkin Seed in Men with Lower Urinary Tract Symptoms due to Benign Prostatic Hyperplasia in the One-Year, Randomized, Placebo-Controlled GRANU Study. Urol Int. 2015;94(3):286-95.

9. Xu ZJ. [Efficacy observation on benign prostatic hyperplasia treated with acupuncture and moxibustion]. Zhongguo Zhen Jiu. 2014;34(3):241-4.

10.Wang Y, Liu B, Yu J, Wu J, Wang J, Liu Z. Electroacupuncture for Moderate and Severe Benign Prostatic Hyperplasia: A Randomized Controlled Trial. PLoS One. 2013;8(4):e59449.

11. Yu JS, Shen KH, Chen WC, Her JS, Hsieh CL. Effects of Electroacupuncture on Benign Prostate Hyperplasia Patients with Lower Urinary Tract Symptoms: A Single-Blinded, Randomized Controlled Trial. Evid Based Complement Alternat Med. 2011;2011:303198. doi: $10.1155 / 2011 / 303198$.

12. Aghajanyan IG Allen S. Positive Response to Thermobalancing Therapy Enabled by Therapeutic Device in Men with Non-Malignant Prostate Diseases: BPH and Chronic Prostatitis. Diseases. 2016;4(2):18.

13. Allen S, Adjani A. Therapeutic Device and Method. US 20110152986 A1. 2009. http://www.google.com/patents/US20110152986.

14. Allen S, Aghajanyan IG. Benign Prostatic Hyperplasia Treatment with New Physiotherapeutic Device, Urol J. 2015 Nov 14;12(5):2371-6.

15. Ben-Zvi T, Hueber PA, Valdivieso R, Azzizi M, Tholomier C, Bienz M, et al. Urological resident exposure to transurethral surgical options for BPH management in 2012-2013: A pan-Canadian survey. Can Urol Assoc J. 2014;8(1-2):54-60.

16. Harvard Med School, Your benign prostatic hyperplasia medication: When to consider a change. Prostate Knowledge. 2007: http://www. harvardprostateknowledge.org/your-benign-prostatic-hyperplasiamedication-when-to-consider-a-change.

17. Irwig MS, Kolukula S. Persistent sexual side effects of finasteride for male pattern hair loss. J Sex Med. 2011 ;8(6):1747-53. doi: 10.1111/j.1743-6109.2011.02255.x.

18. Pearson R, Williams PM. Common Questions about the Diagnosis and Management of Benign Prostatic Hyperplasia. American Family Physician. 2014;90(11):769-774.

19. Elshal AM, Elmansy HM, Elkoushy MA, Elhilali MM. Male sexual function outcome after three laser prostate surgical techniques: a single center perspective. Urology. 2012;80(5):1098-104. doi: 10.1016/j.urology.2012.08.001.

20. Oelke M, Bachmann A, Descazeaud A, Emberton M, Gravas S, Michel MC, et al. EAU Guidelines on the Treatment and Follow-up of Nonneurogenic Male Lower Urinary Tract Symptoms Including Benign Prostatic Obstruction. Eur Urol. 2013;64(1):118-40. doi: 10.1016/j. eururo.2013.03.004.

21. Shimizu S, Tsounapi P, Shimizu T, Honda M, Inoue K, Dimitriadis F, et al. Lower urinary tract symptoms, benign prostatic hyperplasia/benign prostatic enlargement and erectile dysfunction: Are these conditions 
related to vascular dysfunction? Int J Urol. 2014;21(9):856-64.

22. Andersson K, Nomiya M, Yamaguchi O. Chronic Pelvic Ischemia: Contribution to the Pathogenesis of Lower Urinary Tract Symptoms (LUTS): A New Target for Pharmacological Treatment? Low Urin Tract Symptoms. 2015;7(1):1-8. doi: 10.1111/luts.12084.

23. Cohen PG. Abdominal obesity and intra-abdominal pressure: a new paradigm for the pathogenesis of the hypogonadal-obesity-BPH-LUTS connection. Horm Mol Biol Clin Investig. 2012;11(1):317-20. doi: 10.1515/hmbci-2012-0030.
24. Allen S, Aghajanyan I. Thermobalancing Therapy Can Improve the Quality of Life of Patients with Urological Diseases: Chronic Prostatitis, BPH and Kidney Stones. Kidney Urol Res. 2016;1:004.

25. Aghajanyan IG, Allen S. Therapeutic Device Reduces the Prostate Volume in Men with Benign Prostatic Hyperplasia, Easing Lower Urinary Tract Symptoms, Naturally. Kidney Urol Res. 2016;2:005 